\title{
The Number of Matching Equivalent for the Union Graph of Vertices and Cycles
}

\author{
Xiaoling Wang \\ School of Mathematics and Statistics, Qinghai Nationalities University, Xining, China \\ Email: qh_wangxiaoling@163.com
}

How to cite this paper: Wang, X.L. (2021) The Number of Matching Equivalent for the Union Graph of Vertices and Cycles. Applied Mathematics, 12, 471-476. https://doi.org/10.4236/am.2021.126032

Received: May 3, 2021

Accepted: June 21, 2021

Published: June 24, 2021

Copyright $(9) 2021$ by author(s) and Scientific Research Publishing Inc. This work is licensed under the Creative Commons Attribution International License (CC BY 4.0).

http://creativecommons.org/licenses/by/4.0/

\begin{abstract}
For two graphs $G$ and $H$, if $G$ and $H$ have the same matching polynomial, then $G$ and $H$ are said to be matching equivalent. We denote by $\delta(G)$, the number of the matching equivalent graphs of $G$. In this paper, we give $\delta\left(s K_{1} \cup t_{1} C_{9} \cup t_{2} C_{15}\right)$, which is a generation of the results of in [1].
\end{abstract}

\section{Keywords}

Graph, Matching Polynomial, Matching Equivalence

\section{Introduction}

This paper only considers finite undirected simple graphs. Let $G$ be a graph with $n$ vertices. The matching of $G$ means a spanning subgraph of $G$, and each of its connected branches is either an isolated vertex or an isolated edge. $t$-matching means matching that there are $t$ edges. Matching polynomial of graph $G$ is defined as follows in [2]:

$$
\mu(G, x)=\sum_{t \geq 0}(-1)^{t} \alpha_{t}(G) x^{n-2 t}
$$

where $\alpha_{t}(G)$ is number of $t$-matching for $G$.

If graph $G$ and graph $H$ satisfy $\mu(G, x)=\mu(H, x)$, then we say $G$ and $H$ are matching equivalence, denoted as $G \sim H$.

Set $\delta(G)$ which denotes the number of matching equivalent graphs of all different isomorphisms for graph $G$, if $\delta(G)=1$, we say graph $G$ is a unique matching. In [2], the authors gave some elegant properties of matching polynomials, and proved that to find the matching polynomial of a graph is an $N P$-problem. Thus, in [1], the authors studied the number of matching equivalent graphs of some vertices and some cycle-union graphs. It turns out that the problem is not simple. In this paper, we study the number of matching equiva- 
lent for the union graph of vertices and cycles, that is $\delta\left(s K_{1} \cup t_{1} C_{9} \cup t_{2} C_{15}\right)$.

Throughout the paper, $K_{1}$ denotes an isolated vertex, $P_{n}(n \geq 2)$ denotes the path including $n$ vertices; $C_{m}(m \geq 3)$ denotes a cycle that including $m$ vertices; $T_{i, j, k}$ denotes a tree that has only a 3 -degree vertex, three 1 -degree vertex, and the distance between this 3-degree vertex and three 1-degree vertex is $i, j, k$ separately; $D_{n}(n \geq 4)$ denotes a graph that produced by bonding a vertex on a triangle to an end of a path $P_{n-2} ; n G$ denotes disjoint union of $n$ graph $G$.

\section{Preliminaries}

Lemma 2.1 [3] Suppose graph $G$ has $k$ connected component: $G_{1}, G_{2}, \cdots, G_{k}$, then $\mu(G, x)=\prod_{i=1}^{k} \mu\left(G_{i}, x\right)$, the roots of matching polynomials are all real numbers, denote $M(G)$ as the maximum root of $\mu(G, x)$.

Lemma 2.2 [3] Suppose $G$ is connected graph, then $M(G)<2$ if and only if $G \in \Gamma=\left\{K_{1}, P_{n}, T_{1,1, n}, T_{1,2,2}, T_{1,2,3}, T_{1,2,4}, C_{n}, D_{4}\right\}$.

Lemma 2.3 [3] (i) $M\left(C_{m}\right)=M\left(T_{1,1, m-2}\right)=M\left(P_{2 m-1}\right)$.

(ii) $M\left(C_{6}\right)=M\left(T_{1,1,4}\right)=M\left(T_{1,2,2}\right)=M\left(D_{4}\right)=M\left(P_{11}\right)$.

(iii) $M\left(C_{9}\right)=M\left(T_{1,1,7}\right)=M\left(T_{1,2,3}\right)=M\left(P_{17}\right)$.

(iv) $M\left(C_{15}\right)=M\left(T_{1,1,13}\right)=M\left(T_{1,2,4}\right)=M\left(P_{29}\right)$.

Lemma 2.4 [4] [5] (i) The matching equivalent graph of $K_{1} \cup C_{m}(m \neq 6,9,15)$ is $K_{1} \cup C_{m}, T_{1,1, m-2}$.

(ii) The matching equivalent graph of $K_{1} \cup C_{6}$ is: $K_{1} \cup C_{6}, T_{1,1,4}, P_{3} \cup D_{4}$.

(iii) The matching equivalent graph of $K_{1} \cup C_{9}$ is $K_{1} \cup C_{9}, T_{1,1,7}, C_{3} \cup T_{1,2,3}$.

(iv) The matching equivalent graph of $K_{1} \cup C_{15}$ is: $K_{1} \cup C_{15} \sim C_{3} \cup C_{5} \cup T_{1,2,4}$.

Lemma 2.5 [6] (i) $P_{2 m+1} \sim P_{m} \cup C_{m+1},(m \geq 2)$.

(ii) $T_{1,1 n} \sim K_{1} \cup C_{n+2}$.

(iii) $T_{1,2,2} \sim P_{2} \cup D_{4}$.

(iv) $K_{1} \cup C_{6} \sim P_{3} \cup D_{4}$.

(v) $K_{1} \cup C_{9} \sim C_{3} \cup T_{1,2,3}$.

(vi) $K_{1} \cup C_{15} \sim C_{3} \cup C_{5} \cup T_{1,2,4}$.

Lemma 2.6 [1] Suppose $G=s K_{1}$ or $t_{1} C_{m_{1}} \cup \cdots \cup t_{k} C_{m_{k}}$, then $G$ is a unique matching, that is $\delta(G)=1$.

Lemma 2.7 [1] Suppose

$$
G=s K_{1} \cup t_{1} C_{m_{1}} \cup t_{2} C_{m_{2}} \cup \cdots \cup t_{k} C_{m_{k}}, m_{i} \neq 6(i=1,2, \cdots, k),
$$

then all matching equivalent graphs of $G$ do not contain road branches.

Lemma 2.8 [1]

(i) $G=s K_{1} \cup a P_{3} \cup t C_{6}$, then all matching equivalent graphs of $G$ do not contain $P_{11}$ branch and also $T_{1,2,2}$ [7],

(ii) $\delta\left(s K_{1} \cup a P_{3} \cup t C_{6}\right)=\delta\left(s K_{1} \cup t C_{6}\right)$.

Lemma 2.9 [1] [7] If $m \neq 6,9,15$, then

$$
\delta\left(s K_{1} \cup t C_{6}\right)=\min \{s, t\}+1 .
$$

Lemma 2.10 [1] [8] If $m_{i} \neq 6,9,15(i=1,2)$, then

$$
\delta\left(s K_{1} \cup t_{1} C_{m_{1}} \cup t_{2} C_{m_{2}}\right)=\sum_{i=0}^{r} \min \left\{s-i, t_{1}\right\}+r+1 \text {, where }
$$




$$
r=\min \left\{s, t_{2}\right\}
$$

\section{Main Results}

Lemma 3.1 $\delta\left(s K_{1} \cup t_{1} C_{3} \cup t_{2} C_{5} \cup t_{3} C_{9}\right)=\sum_{j=0}^{r} \sum_{i=j}^{r} \delta\left(s-i, t_{1}+(i-j), t_{2}\right)$, where

$$
r=\min \left\{s, t_{3}\right\} .
$$

Proof. For simplicity, denote $\delta\left(s K_{1} \cup t_{1} C_{3} \cup t_{2} C_{5} \cup t_{3} C_{9}\right)=\delta\left(s, t_{1}, t_{2}, t_{3}\right)$.

Set $H \sim s K_{1} \cup t_{1} C_{3} \cup t_{2} C_{5} \cup t_{3} C_{6}$, by lemma 2.3 (iii) and lemma 2.7, we know $H$ contains connected component $C_{9}, T_{1,1,7}$ or $T_{1,2,3}$.

1) If $H$ contains $C_{9}$, by $H=C_{9} \cup H_{2} \sim s K_{1} \cup t_{1} C_{3} \cup t_{2} C_{5} \cup t_{3} C_{9}$, we know $H_{2} \sim s K_{1} \cup t_{1} C_{3} \cup t_{2} C_{5} \cup\left(t_{3}-1\right) C_{9}$.

Such $\mathrm{H}_{2}$ has a total of $\delta\left(s, t_{1}, t_{2}, t_{3}-1\right)$.

2) If $H$ contains $T_{1,1,7}$, by $H=T_{1,1,7} \cup H_{2} \sim s K_{1} \cup t_{1} C_{3} \cup t_{2} C_{5} \cup t_{3} C_{9}$ and lemma 2.5 (ii), we know

$$
H_{2} \sim(s-1) K_{1} \cup t_{1} C_{3} \cup t_{2} C_{5} \cup\left(t_{3}-1\right) C_{9},
$$

Such $H_{2}$ has a total of $\delta\left(s-1, t_{1}, t_{2}, t_{3}-1\right)$.

3) If $H$ contains $T_{1,2,3}$, by $H=T_{1,2,3} \cup H_{2} \sim s K_{1} \cup t_{1} C_{3} \cup t_{2} C_{5} \cup t_{3} C_{9}$ and lemma 2.5(v), we get

$$
H_{2} \sim(s-1) K_{1} \cup\left(t_{1}+1\right) C_{3} \cup t_{2} C_{5} \cup\left(t_{3}-1\right) C_{9},
$$

Such $H_{2}$ has a total of $\delta\left(s-1, t_{1}+1, t_{2}, t_{3}-1\right)$.

4) If $H$ contains $C_{9}$ and $T_{1,1,7}$ simultaneously, such $H_{2}$ has a total of $\delta\left(s-1, t_{1}, t_{2}, t_{3}-2\right)$.

5) If $H$ contains $C_{9}$ and $T_{1,2,3}$ simultaneously, such $H_{2}$ has a total of $\delta\left(s-1, t_{1}+1, t_{2}, t_{3}-2\right)$.

6) If $H$ contains $T_{1,1,7}$ and $T_{1,2,3}$ simultaneously, such $H_{2}$ has a total of $\delta\left(s-2, t_{1}+1, t_{2}, t_{3}-2\right)$.

7) If $H$ contains $C_{9}, T_{1,1,7}$ and $T_{1,2,3}$ simultaneously, such $H_{2}$ has a total of $\delta\left(s-2, t_{1}+1, t_{2}, t_{3}-3\right)$.

Thus,

$$
\begin{aligned}
\delta\left(s, t_{1}, t_{2}, t_{3}\right)= & \delta\left(s, t_{1}, t_{2}, t_{3}-1\right)+\delta\left(s-1, t_{1}, t_{2}, t_{3}-1\right)+\delta\left(s-1, t_{1}+1, t_{2}, t_{3}-1\right) \\
& -\delta\left(s-1, t_{1}, t_{2}, t_{3}-2\right)-\delta\left(s-1, t_{1}+1, t_{2}, t_{3}-2\right) \\
& -\delta\left(s-2, t_{1}+1, t_{2}, t_{3}-2\right)+\delta\left(s-2, t_{1}+1, t_{2}, t_{3}-3\right)
\end{aligned}
$$

Then,

$$
\begin{aligned}
& \delta\left(s, t_{1}, t_{2}, t_{3}\right)-\delta\left(s-1, t_{1}, t_{2}, t_{3}-1\right)-\delta\left(s-1, t_{1}+1, t_{2}, t_{3}-1\right) \\
& +\delta\left(s-2, t_{1}+1, t_{2}, t_{3}-2\right) \\
& =\delta\left(s, t_{1}, t_{2}, t_{3}-1\right)-\delta\left(s-1, t_{1}, t_{2}, t_{3}-2\right)-\delta\left(s-1, t_{1}+1, t_{2}, t_{3}-2\right) \\
& +\delta\left(s-2, t_{1}+1, t_{2}, t_{3}-3\right)
\end{aligned}
$$

Repeat the application of the above formula, we obtain 


$$
\begin{aligned}
& \delta\left(s, t_{1}, t_{2}, t_{3}\right)-\delta\left(s-1, t_{1}, t_{2}, t_{3}-1\right)-\delta\left(s-1, t_{1}+1, t_{2}, t_{3}-1\right) \\
& +\delta\left(s-2, t_{1}+1, t_{2}, t_{3}-2\right) \\
& =\delta\left(s, t_{1}, t_{2}, 2\right)-\delta\left(s-1, t_{1}, t_{2}, 1\right)-\delta\left(s-1, t_{1}+1, t_{2}, 1\right)+\delta\left(s-2, t_{1}+1, t_{2}, 0\right) \\
& =\delta\left(s, t_{1}, t_{2}, 1\right)-\delta\left(s-1, t_{1}, t_{2}, 0\right)-\delta\left(s-1, t_{1}+1, t_{2}, 0\right) \\
& =\delta\left(s, t_{1}, t_{2}, 0\right)=\delta\left(s, t_{1}, t_{2}\right)
\end{aligned}
$$

Thus,

$$
\begin{aligned}
& \delta\left(s, t_{1}, t_{2}, t_{3}\right)-\delta\left(s-1, t_{1}, t_{2}, t_{3}-1\right) \\
& =\delta\left(s-1, t_{1}+1, t_{2}, t_{3}-1\right)-\delta\left(s-2, t_{1}+1, t_{2}, t_{3}-2\right)+\delta\left(s, t_{1}, t_{2}\right) \\
& =\delta\left(s-2, t_{1}+2, t_{2}, t_{3}-2\right)-\delta\left(s-3, t_{1}+2, t_{2}, t_{3}-3\right) \\
& +\delta\left(s-1, t_{1}+1, t_{2}\right)+\delta\left(s-1, t_{1}+1, t_{2}\right) \\
& =\cdots=\sum_{i=0}^{r} \delta\left(s-i, t_{1}+i, t_{2}\right) \\
& \delta\left(s, t_{1}, t_{2}, t_{3}\right)-\delta\left(s-1, t_{1}, t_{2}, t_{3}-1\right)=\sum_{i=0}^{r} \delta\left(s-i, t_{1}+i, t_{2}\right) \\
& \delta\left(s-1, t_{1}, t_{2}, t_{3}-1\right)-\delta\left(s-2, t_{1}, t_{2}, t_{3}-2\right)=\sum_{i=1}^{r} \delta\left(s-i, t_{1}+(i-1), t_{2}\right) \\
& \text {... } \\
& \delta\left(s-(r-1), t_{1}, t_{2}, t_{3}-(r-1)\right)-\delta\left(s-r, t_{1}, t_{2}, t_{3}-r\right) \\
& =\sum_{i=r-1}^{r} \delta\left(s-i, t_{1}+i-(r-1), t_{2}\right) \\
& \delta\left(s-r, t_{1}, t_{2}, t_{3}-r\right)=\sum_{i=r}^{r} \delta\left(s-i, t_{1}+(i-r), t_{2}\right) \quad(r+1)
\end{aligned}
$$

Add (1), (2), $\cdots,(r+1)$ together, we get

$$
\delta\left(s, t_{1}, t_{2}, t_{3}\right)=\sum_{j=0}^{r} \sum_{i=j}^{r} \delta\left(s-i, t_{1}+(i-j), t_{2}\right) .
$$

\section{Theorem 3.1}

$$
\delta\left(s K_{1} \cup t_{1} C_{9} \cup t_{2} C_{15}\right)=\sum_{j=0}^{r} \sum_{i=j}^{r} \delta\left(s-i, i-j, i-j, t_{1}\right) .
$$

Proof. For simplicity, denote

$$
\delta\left(s K_{1} \cup t C_{3} \cup t^{\prime} C_{5} \cup t_{1} C_{9} \cup t_{2} C_{15}\right)=\delta\left(s, t, t^{\prime} t_{1}, t_{2}\right) .
$$

Suppose $H \sim G$, by lemma 2.3(iv) and lemma 2.7, we know $H$ contains connected component $C_{15}, T_{1,1,13}$ or $T_{1,2,4}$.

1) If $H$ contains $C_{15}$, by $H=C_{15} \cup H_{2} \sim s K_{1} \cup t_{1} C_{9} \cup t_{2} C_{15}$ we know $H_{2} \sim s K_{1} \cup t_{1} C_{9} \cup\left(t_{2}-1\right) C_{15}$. Such $H_{2}$ has a total of $\delta\left(s, 0,0, t_{1}, t_{2}-1\right)$.

2) If $H$ contains $T_{1,1,13}$, by $H=T_{1,1,13} \cup H_{2} \sim G=s K_{1} \cup t_{1} C_{9} \cup t_{2} C_{15}$ and lemma 2.5(ii), we get $H_{2} \sim(s-1) K_{1} \cup t_{1} C_{9} \cup\left(t_{2}-1\right) C_{15}$. Such $H_{2}$ has a total of $\delta\left(s-1,0,0, t_{1}, t_{2}-1\right)$.

3) If $H$ contains $T_{1,2,4}$, by $H=T_{1,2,4} \cup H_{2} \sim G=s K_{1} \cup t_{1} C_{9} \cup t_{2} C_{15}$ and lemma 2.5(vi), we get $H_{2} \sim(s-1) K_{1} \cup C_{3} \cup C_{5} \cup t_{1} C_{9} \cup\left(t_{2}-1\right) C_{15}$, such $H_{2}$ has a total of $\delta\left(s-1,1,1, t_{1}, t_{2}-1\right)$. 
4) If $H$ contains $C_{15}$ and $T_{1,1,13}$, such $H_{2}$ has a total of $\delta\left(s-1,0,0, t_{1}, t_{2}-2\right)$.

5) If $H$ contains $C_{15}$ and $T_{1,2,4}$, such $H_{2}$ has a total of $\delta\left(s-1,1,1, t_{1}, t_{2}-2\right)$.

6) If $H$ contains $T_{1,1,13}$ and $T_{1,2,4}$, such $H_{2}$ has a total of $\delta\left(s-2,1,1, t_{1}, t_{2}-2\right)$.

7) If $H$ contains $C_{15}, T_{1,1,13}$ and $T_{1,2,4}$, such $H_{2}$ has a total of $\delta\left(s-2,1,1, t_{1}, t_{2}-3\right)$.

Then

$$
\begin{aligned}
\delta\left(s, 0,0, t_{1}, t_{2}\right)= & \delta\left(s, 0,0, t_{1}, t_{2}-1\right)+\delta\left(s-1,0,0, t_{1}, t_{2}-1\right) \\
& +\delta\left(s-1,1,1, t_{1}, t_{2}-1\right)-\delta\left(s-1,0,0, t_{1}, t_{2}-2\right) \\
& -\delta\left(s-1,1,1, t_{1}, t_{2}-2\right)-\delta\left(s-2,1,1, t_{1}, t_{2}-2\right) \\
& +\delta\left(s-2,1,1, t_{1}, t_{2}-3\right)
\end{aligned}
$$

Thus,

$$
\begin{aligned}
& \delta\left(s, 0,0, t_{1}, t_{2}\right)-\delta\left(s-1,0,0, t_{1}, t_{2}-1\right)-\delta\left(s-1,1,1, t_{1}, t_{2}-1\right) \\
& +\delta\left(s-2,1,1, t_{1}, t_{2}-2\right) \\
& =\delta\left(s, 0,0, t_{1}, t_{2}-1\right)-\delta\left(s-1,0,0, t_{1}, t_{2}-2\right)-\delta\left(s-1,1,1, t_{1}, t_{2}-2\right) \\
& +\delta\left(s-2,1,1, t_{1}, t_{2}-3\right)
\end{aligned}
$$

Repeat the application of the above formula, we obtain

$$
\begin{aligned}
& \delta\left(s, 0,0, t_{1}, t_{2}\right)-\delta\left(s-1,0,0, t_{1}, t_{2}-1\right)-\delta\left(s-1,1,1, t_{1}, t_{2}-1\right) \\
& +\delta\left(s-2,1,1, t_{1}, t_{2}-2\right) \\
& =\delta\left(s, 0,0, t_{1}, 2\right)-\delta\left(s-1,0,0, t_{1}, 1\right)-\delta\left(s-1,1,1, t_{1}, 1\right)+\delta\left(s-2,1,1, t_{1}, 0\right) \\
& =\delta\left(s, 0,0, t_{1}, 1\right)-\delta\left(s-1,0,0, t_{1}, 0\right)-\delta\left(s-1,1,1, t_{1}, 0\right) \\
& =\delta\left(s, 0,0, t_{1}\right)
\end{aligned}
$$

So,

$$
\begin{aligned}
& \delta\left(s, 0,0, t_{1}, t_{2}\right)-\delta\left(s-1,0,0, t_{1}, t_{2}-1\right) \\
& =\delta\left(s-1,1,1, t_{1}, t_{2}-1\right)-\delta\left(s-2,1,1, t_{1}, t_{2}-2\right)+\delta\left(s, 0,0, t_{1}\right) \\
& =\delta\left(s-2,2,2, t_{1}, t_{2}-2\right)-\delta\left(s-3,2,2, t_{1}, t_{2}-3\right) \\
& +\delta\left(s, 0,0, t_{1}\right)+\delta\left(s-1,1,1, t_{1}\right) \\
& =\delta\left(s-3,3,3, t_{1}, t_{2}-3\right)-\delta\left(s-4,3,3, t_{1}, t_{2}-4\right)+\delta\left(s, 0,0, t_{1}\right) \\
& +\delta\left(s-1,1,1, t_{1}\right)+\delta\left(s-2,2,2, t_{1}\right) \\
& =\cdots=\sum_{i=0}^{r} \delta\left(s-i, i, i, t_{1}\right) \\
& \delta\left(s, 0,0, t_{1}, t_{2}\right)-\delta\left(s-1,0,0, t_{1}, t_{2}-1\right)=\sum_{i=0}^{r} \delta\left(s-i, i, i, t_{1}\right) \\
& \delta\left(s-1,0,0, t_{1}, t_{2}-1\right)-\delta\left(s-2,0,0, t_{1}, t_{2}-2\right)=\sum_{i=0}^{r} \delta\left(s-i, i-1, i-1, t_{1}\right) \\
& \text {... } \\
& \delta\left(s-(r-1), 0,0, t_{1}, t_{2}-(r-1)\right)-\delta\left(s-r, 0,0, t_{1}, t_{2}-r\right) \\
& =\sum_{i=r-1}^{r} \delta\left(s-i, i-(r-1), i-(r-1), t_{1}\right)
\end{aligned}
$$


$\delta\left(s-r, 0,0, t_{1}, t_{2}-r\right)=\sum_{i=r}^{r} \delta\left(s-i, i-r, i-r, t_{1}\right) \quad\left(r+1^{\prime \prime}\right)$

Add $\left(1^{\prime}\right),\left(2^{\prime \prime}\right), \cdots,\left(r+1^{\prime \prime}\right)$ together, we get:

$$
\delta\left(s, 0,0, t_{1}, t_{2}\right)=\sum_{j=0}^{r} \sum_{i=j}^{r} \delta\left(s-i, i-j, i-j, t_{1}\right) .
$$

Characterizing all graphs determined by a graph polynomial is an important subject in algebraic graph theory, among them, matching polynomial is considered to be a better algebraic tool. It is NP difficult to completely characterize the matched equivalent graphs of a class of graphs. In this paper, we study the number of matching equivalent graphs of some points and some cycli-union graphs, that is to say we calculate

$\delta\left(s K_{1} \cup t_{1} C_{9} \cup t_{2} C_{15}\right)=\sum_{j=0}^{r} \sum_{i=j}^{r} \delta\left(s-i, i-j, i-j, t_{1}\right)$.

\section{Acknowledgements}

This work is supported by the National Natural Science Foundation of China (No. 11561056), the Natural Science Foundation of Qinghai Province (2016ZJ-914), Teaching Reform Research Project of Qinghai Nationalities University (2021-JYQN-005).

\section{Conflicts of Interest}

The author declares no conflicts of interest regarding the publication of this paper.

\section{References}

[1] Ma, H.C. and Wang, X.L. (2006) Matching Equivalent Graph Class of Point-Circle Union Graph. Journal of Northeast Normal University (Natural Science Edition), 4, 36-40.

[2] Godsil, C.D. (1993) Algebraic Combinatorics. Chapman and Hall, New York, London.

[3] Ma, H.C. (2000) Matching Equivalence Classes of Two Types of Graphs. Journal of Mathematical Study, 2, 218-222.

[4] Ma, H.C. (2003) Matches the Matching Equivalence Class of Graphs Whose Maximum Root Is Less than 2. Journal of Systems Science and Mathematical Sciences, 3, 337-342.

[5] Ma, H.C. and Li, Y.K. (2016) The Matching Equivalence Graphs with the Maximam Matching Root Less than or Equal to 2. Applied Mathematics, 7, 920-926. https://doi.org/10.4236/am.2016.79082

[6] Guo, Z.Y. and Yu, Y.S. (1989) On the Matching Uniqueness of Two Kinds of Graphs. Mathematica Applicata, 2, 25-32.

[7] Ma, H.C. (2017) A Characterization of Graphs with Rank More than 5. Applied Mathematics, 8, 26-34. https://doi.org/10.4236/am.2017.81003

[8] Ma, H.C. (2017) The Energy and Operations of Graphs. Advances in Pure Mathematics, 7, 345-351. https://doi.org/10.4236/apm.2017.76021 